\title{
Calmodulin Expression Distinguishes the Smooth Muscle Cell Population of Human Carotid Plaque
}

\author{
Matteo Coen ${ }^{* \dagger}$ Giovanna Marchetti, ${ }^{\ddagger}$ Patricia M. Palagi, ${ }^{\S}$ Carlotta Zerbinati, ${ }^{\ddagger}$ Giuseppe Guastella, ${ }^{\ddagger}$ Teresa Gagliano, ${ }^{\natural}$ \\ Francesco Bernardi, " Francesco Mascoli, ${ }^{\dagger}$ and Marie-Luce Bochaton-Piallat*
}

\begin{abstract}
From the Department of Pathology and Immunology, * Faculty of Medicine, University of Geneva, Geneva, Switzerland; the Operative Unit of Vascular and Endovascular Surgery, ${ }^{\dagger}$ S. Anna University-Hospital, Ferrara, Italy; the Department of Biomedical and Specialty Surgical Sciences, ${ }^{\ddagger}$ the Section of Endocrinology, "Department of Medical Sciences, and the Department of Life Sciences and Biotechnology," University of Ferrara, Ferrara, Italy; and the Proteome Informatics Group,${ }^{\S}$ Swiss Institute of Bioinformatics, Geneva, Switzerland
\end{abstract}

Accepted for publication June 3, 2013.

Address correspondence to Marie-Luce Bochaton-Piallat, Ph.D., Department of Pathology and Immunology, Faculty of Medicine, University of Geneva-CMU, 1, rue Michel Servet, 1211 Geneva 4, Switzerland. E-mail: Marie-Luce. Piallat@unige.ch.

\begin{abstract}
Several observations suggest the expansion of a distinct medial smooth muscle cell (SMC) subset in atherosclerosis and restenosis. We characterized the phenotypic features of SMC subsets in cultures derived from human carotid endarterectomy specimens. Specimens comprised an undiseased portion (thin intimal thickening with the underlying media) and a diseased portion (atherosclerotic plaque with the underlying media). From plaque tissues of the diseased portion, only macrophage-derived foam cells were retrieved. From medial tissues, two SMC phenotypes were isolated: large SMCs (flat with a monolayered growth pattern, from the undiseased portion) and small SMCs (fusiform and growing in multilayers, from the undiseased and diseased portions after co-culture with macrophagederived foam cells). Small SMCs displayed higher proliferative and migratory activities and were less differentiated than large SMCs. Proteomic analysis showed that calmodulin was predominant in small SMCs. Co-culture of large SMCs with macrophage-derived foam cells induced a transition to the small phenotype with increased calmodulin expression. The calmodulin inhibitor W-7 decreased the proliferation of small SMCs and prevented the large to small phenotypic transition. In vivo, calmodulin was markedly expressed in SMCs of atherosclerotic plaques and was barely detectable in the media. Macrophage-derived foam cells promote selective migration from the media of atheromaprone SMCs characterized by calmodulin overexpression. Further studies of small SMCs could be instrumental in understanding atherosclerosis pathogenesis and in planning therapeutic strategies. (Am J Pathol 2013, 183: 996-1009; http://dx.doi.org/10.1016/j.ajpath.2013.06.006)
\end{abstract}

Atherosclerosis is the major cause of cardiovascular diseases, including coronary and peripheral artery occlusive diseases, stroke, and renovascular hypertension; it represents the leading cause of mortality and morbidity in developed countries. Moreover, restenosis stands as a major limitation of revascularization procedures, particularly in peripheral arteries. ${ }^{1}$

It is well accepted that smooth muscle cells (SMCs) are a major component of atherosclerotic and restenotic lesions, where they contribute substantially to lumen narrowing. ${ }^{2}$ Despite progress in understanding arterial SMC biology, several questions remain open, such as the relative contribution of a distinct SMC subpopulation to atheroma or restenosis formation and the origin of the stimuli involved in SMC migration from the media to the intima. In this respect, although different factors (eg, cytokines ${ }^{3}$ and oxidized lowdensity lipoproteins ${ }^{4}$ ) have been shown to promote SMC proliferation and migration, none of them seems to act selectively on a particular SMC subpopulation.

After the seminal observation by Benditt and Benditt, ${ }^{5}$ later confirmed by Murry et al, ${ }^{6}$ that plaque SMCs have a monoclonal or oligoclonal derivation from media precursors, thus

Supported by Swiss National Science Foundation grants 310030_130700/1 and 310030_146790/1; Fondation Artères, Fondation Gustave et Simone Prévot, Jubiläumsstiftung Swiss life, Novartis Foundation for Medicine and Biology grant 06C82; and Fondazione Cassa di Risparmio di Ferrara and Ministero Istruzione Università Ricerca. M.C. was partially supported by the Ernst and Lucie Schmidheiny Foundation and by a training (research) fellowship from the Fondazione Italiana Sclerosi Multipla (Cod.297/10/F1). 
supporting the assumption that arterial SMCs are heterogeneous, distinct SMC populations have been isolated and characterized in vitro in several animal models, including rat, ${ }^{7-9}$ cow, ${ }^{10}$ and pig. ${ }^{11}$ Most of these populations exhibit a phenotype characterized by high-proliferative, migratory, and proteolytic activities and a poor level of differentiation, features compatible with an atheroma-prone phenotype. ${ }^{12}$ Different markers of this phenotype have been described according to the experimental model; some of them have proved to be relevant to the human situation. $^{13,14}$ Arterial SMC subpopulations have also been isolated from humans ${ }^{15-19}$; however, the importance of a particular SMC subpopulation in atherogenesis has not been demonstrated, possibly owing to limited availability and lack of experimental standardization.

The aim of this study was to reproducibly isolate and thoroughly characterize human arterial SMC subpopulations to evaluate their role in atheroma formation. We demonstrate that two distinct SMC phenotypes can be isolated from human carotid endarterectomy (CEA) specimens: large and small SMCs. Small SMCs are selectively attracted by atheromatous plaque macrophage-derived foam cells and exhibit features consistent with the atheroma-prone phenotype. By the proteomic approach, we identified calmodulin $(\mathrm{CaM})$ as a marker of the small SMC phenotype; moreover, we showed that inhibition of CaM decreases small SMC proliferative activity. In vivo, CaM seems to be expressed by a subpopulation of SMCs in atherosclerotic plaques, thus suggesting a role in atheroma or restenosis development.

\section{Materials and Methods}

\section{Specimen Collection}

A total of 41 CEA specimens, which were used for the different experiments, were obtained at surgery from consecutive patients (asymptomatic) who underwent conventional CEA for extracranial high-grade internal carotid artery stenosis ( $>70 \%$ luminal narrowing for symptomatic patients according to the North American Symptomatic Carotid Endarterectomy Trial criteria for carotid stenosis $)^{20}$ by the same surgeon (F.M.). Restenotic lesions after CEA were not included. The study was approved by the ethic committee of the S. Anna University-Hospital (Ferrara, Italy), and written informed consent was obtained from all the patients. Arteriotomy was performed on the common carotid artery and extended to the internal carotid artery. A dissector was used to separate the outer media from the underlying adventitia. The specimen was then removed intact as a single piece through an incision along the lateral aspect of the carotid artery and was saved for cell culture and histologic analysis. Each sample was cut transversally at the bifurcation; only the portion toward the aortic arch and consisting of a complete cast of the common carotid artery was used. This segment was further cut into two portions: a proximal, undiseased portion (UP) and a distal, diseased portion (DP) characterized by diffuse atherosclerotic lesions. Representative tissue blocks were obtained from each segment and were processed for histologic and immunohistochemical analyses or for cell culture.

\section{Antibodies}

The following primary mouse monoclonal antibodies were used: IgG2a recognizing $\alpha$-smooth muscle actin $(\alpha$-SMA, clone $1 \mathrm{~A} 4)^{21}$; IgG1 recognizing vimentin (clone V9; Dako, Glostrup, Denmark); IgG1 recognizing smoothelin A and B (clone R4A) ${ }^{22}$; IgM recognizing S100A4 (clone 4B4) ${ }^{13}$; IgG1 recognizing CaM (clone CaM85; Zymed-Invitrogen, Carlsbad, CA); IgG1 recognizing CD68, a marker of macrophages (clone KP1; Dako); IgG1 recognizing CD3, a marker of T lymphocytes (clone F7.2.38; Dako); IgG1 recognizing CD79, a marker of B-lymphocytes (clone JCB117; Dako); and IgG1 recognizing bromodeoxyuridine (BrdU) (clone Bu20a; Dako). Rabbit polyclonal IgGs recognizing SMMHC types 1 and 2 (BT-562; Biomedical Technologies Inc., Stoughton, MA) were also used.

\section{Histologic and Immunohistochemical Analyses}

Representative specimens of the UP $(n=5)$ and DP $(n=$ 10) were fixed with $4 \%$ buffered formalin and were embedded in paraffin. Sections, $3 \mu \mathrm{m}$ thick, were stained with $H \& E$ and Miller stains. Immunostaining for $\alpha$-SMA, SMMHCs, smoothelin, S100A4, CD68, CD3, and CD79 was performed on adjacent sections. Before using the first antibody, immunoreactivity was intensified by microwave treatment $(750 \mathrm{~W}, 5$ minutes $)$ in citrate buffer $(10 \mathrm{mmol} / \mathrm{L}$, pH 6.0) for $\alpha$-SMA and CD68; by pressure cooker treatment (3 minutes) in citrate buffer for SMMHCs, smoothelin, and S100A4; and by Pascal pressure cooker (Dako) treatment at $125^{\circ} \mathrm{C}$ for 30 minutes for CD3 and CD79. Goat anti-mouse or anti-rabbit biotinylated antibodies (Dako) were used as secondary antibodies. The presence of the specific proteins was revealed by means of the streptavidin-biotin peroxidase complex, and staining was visualized using either the 3,3'diaminobenzidine (SMMHCs, smoothelin, S100A4, and $\mathrm{CaM}$ ) or the 3-amino-9-ethyl-carbazole (CD68, CD3, and CD 79) chromophore. In one case ( $\alpha$-SMA), an alkaline phosphatase complex method was used (EnVision system; Dako). Hemalun was used as counterstaining. Images were taken by means of an Axioskop microscope equipped with Plan-Neofluar $10 \times / 0.3$ and $20 \times / 0.3$ objectives (Carl Zeiss MicroImaging $\mathrm{GmbH}$, Jena, Germany).

\section{SMC Culture and Co-Culture Experiments}

The specimens retrieved at surgery were conserved in cold sterile RPMI 1640 medium supplemented with 2\% HEPES, $100 \mathrm{U} / \mathrm{mL}$ of penicillin, $100 \mu \mathrm{g} / \mathrm{mL}$ of streptomycin, and $2 \mathrm{mmol} / \mathrm{L}$ L-glutamine (Gibco-Invitrogen, Carlsbad, CA). From the UP $(n=25)$, media tissues were obtained after 
intimal and abluminal scraping to remove endothelial and adventitial tissue, respectively, and were cut into $3 \times 3$ - $\mathrm{mm}$ pieces. From the DP $(n=23)$, after gentle scraping of the luminal surface, media and plaque were mechanically separated from each other using tweezers and were cut into $3 \times 3-\mathrm{mm}$ pieces. Tissue dissection was checked histologically. The abluminal side of the explants was carefully placed in contact with the culture dish (5 to 10 tissue pieces for the UP and 15 to 20 tissue pieces for the DP per $60-\mathrm{mm}$ dish). In addition, co-culture of media and plaque pieces was performed by using the Transwell system (24-mmdiameter Transwell clear polyester membrane, $0.4-\mu \mathrm{m}$ pore size; Costar, Corning Inc., Corning, NY). Thirty media pieces from the UP or DP and 30 plaque pieces from the DP were plated separately into either the upper or lower compartment of the system. As control, the tissue pieces of the same origin were co-cultured in the upper and lower compartments. RPMI 1640 medium plus 10\% fetal bovine serum (FBS; Gibco-Invitrogen) was added dropwise. Medium was changed 7 days after plating and every 3 days thereafter. The percentage of tissue explants producing cell outgrowth (ratio of explants with cell outgrowth/total number of seeded explants) and the time requested for emergence of the first cells and for growth to confluence were recorded. Explanted tissue pieces were removed 7 to 10 days after the first SMCs appeared. Cells were passed by a 1:2 split ratio. Twenty populations isolated from the UP and 20 isolated from the DP were studied between the fifth and tenth passage. Plaque-derived conditioned medium (CM) was collected from cultures of DP plaque pieces 72 hours after plating. CM was diluted 1:1 (v/v) with fresh medium supplemented with 10\% FBS and added to passaged SMCs for 72 hours.

\section{Cell Proliferation and Migration}

SMCs ( $n=4$ for each phenotype) were plated at a density of 110 cells $/ \mathrm{mm}^{2}$ in RPMI 1640 medium supplemented with $10 \%$ FBS. Medium was changed every 3 days. In another set of experiments, medium was changed 24 hours after plating, and large SMCs $(n=2)$ were incubated alone or combined with $10 \mathrm{ng} / \mathrm{mL}$ of platelet-derived growth factor (PDGF)-BB (Fluka/Sigma-Aldrich, Buchs, Switzerland). Medium was changed after 3 days, and cells were treated for 6 days. For reversal experiments, cells were treated with PDGF-BB for 6 days, and then the medium was changed for RPMI 1640 medium supplemented with 10\% FBS for 6 days. In both experiments, cells were counted using a hemocytometer. The results are expressed as the percentage of growth rate.

Cell migration was assessed by means of collagen gel invasion assay. SMCs $(n=3$ for the small phenotype and $n=4$ for the large phenotype) were seeded at a density of 200 cells $/ \mathrm{mm}^{2}$ in RPMI 1640 medium plus $10 \%$ FBS on the surface of type I mouse tail collagen gels $(1 \mathrm{mg} / \mathrm{mL}$ in $0.1 \%$ acetic acid). ${ }^{11}$ Moreover, to test the influence of PDGF-BB on large SMC migration, collagen gel-seeded SMCs $(n=4)$ were incubated alone or combined with $10 \mathrm{ng} / \mathrm{mL}$ of PDGF-BB (Fluka/Sigma-Aldrich). Experiments were performed in triplicate.

After 7 days, cell invasion was assessed by counting the number of cells in six randomly selected phase-contrast fields per condition. Each field measured $3.14 \mathrm{~mm}^{2}$ and was obtained at $50-\mu \mathrm{m}$ intervals along the collagen gel from the top $(0 \mu \mathrm{m})$ to $700 \mu \mathrm{m}$ beneath the surface monolayer.

\section{Immunofluorescence Staining}

SMCs ( $n=13$ for the large phenotype and $n=14$ for the small phenotype) were fixed for 20 minutes using $1 \%$ paraformaldehyde in RPMI 1640 medium with 2\% HEPES and then were rinsed in PBS. They were further incubated for 5 minutes in methanol at $-20^{\circ} \mathrm{C}^{23}$ and were double stained with anti- $\alpha$-SMA and either anti-S100A4 or anti$\mathrm{CaM}$; alternatively, they were fixed in ethanol for 30 seconds and were stained with anti-SMMHCs. Macrophages $(n=3)$ were fixed in paraformaldehyde as above. They were incubated with Oil Red O solution [0.3\% Oil Red O (Fluka/Sigma-Aldrich), 60\% isopropanol, and 40\% water] or fixed for 5 minutes in methanol at $-20^{\circ} \mathrm{C}$ and stained with anti-CD68. Sections, $3 \mu \mathrm{m}$ thick, from formalin-fixed, paraffin-embedded DP specimens were double stained with anti- $\alpha$-SMA and anti-CaM. Before using the first antibody, immunoreactivity was intensified by microwave treatment ( $250 \mathrm{~W}$ for 20 minutes) in $10 \mathrm{mmol} / \mathrm{L}$ Tris containing 1 $\mathrm{mmol} / \mathrm{L}$ EDTA, $\mathrm{pH} 9.1{ }^{23}$ Rhodamine-conjugated goat antimouse IgM, fluorescein-conjugated goat anti-mouse IgG2a or $\mathrm{IgG1}$, and rhodamine-conjugated goat anti-rabbit IgG were used as secondary antibodies (Southern Diagnostic Laboratories, Birmingham, AL). Nuclei were stained with DAPI (Fluka/Sigma-Aldrich). Slides were mounted in buffered polyvinyl alcohol. SMC and DP specimen images were taken by means of an Axioskop 2 microscope (Carl Zeiss MicroImaging $\mathrm{GmbH}$ ) equipped with oil immersion Plan-Neofluar $40 \times / 1.4$ and $63 \times / 1.25$ objectives and a highsensitivity, high-resolution digital color camera (Axiocam, Carl Zeiss MicroImaging GmbH). Macrophage images were acquired by using a confocal laser scan fluorescenceinverted microscope (LSM 510 Meta; Carl Zeiss MicroImaging $\mathrm{GmbH}$ ) equipped with a helium-neon laser (excitation wavelength at $543 \mathrm{~nm}$ ) and a diode laser (excitation wavelength at $405 \mathrm{~nm}$ ) through an oil immersion Plan-Neofluar $40 \times / 1.30$ objective.

For BrdU detection, cells were fixed with methanol at $-20^{\circ} \mathrm{C}$ for 5 minutes. After washing in PBS, they were incubated for 20 minutes in $1 \mathrm{~mol} / \mathrm{L} \mathrm{HCl}$ followed by 0.1 $\mathrm{mol} / \mathrm{L}$ anhydrous disodium tetraborate $(\mathrm{pH}$ 8.5; Merck, Darmstadt, Germany) for 5 minutes. The incorporated BrdU was detected using anti-BrdU followed by Alexa Fluor 488-conjugated goat anti-mouse IgG (Molecular Probes Inc., Eugene, OR). Nuclei were stained with DAPI. Slides were mounted in buffered polyvinyl alcohol (Fluka/SigmaAldrich). The percentage of BrdU-positive cells was counted 
by using an Axioskop 2 microscope (Carl Zeiss MicroImaging $\mathrm{GmbH}$ ) at $\times 40$ magnification in 10 different fields for each sample.

\section{SMC Area Measurement}

Trypsinized SMCs ( $n=4$ for each phenotype) were cytocentrifuged using a cytospin (Cytospin II; Shandon, Pittsburgh, PA) on glass slides at a density of $10^{4}$ cells per slide. The preparations were fixed as described for immunofluorescence staining. Phase-contrast images of the cells were recorded using a Zeiss Axiovert 35 photomicroscope at $\times 40$ magnification (Carl Zeiss MicroImaging $\mathrm{GmbH}$ ), and the MetaMorph imaging system (Molecular Devices Inc., Sunnyvale, CA) was used for digital quantification. The mean cell area was calculated in 10 random fields.

\section{Protein Extraction, Electrophoresis, and Western Blot Analysis}

SMCs $(n=10$ for the small phenotype, $n=13$ for the large phenotype, and $n=2$ for the large phenotype treated with PDGF-BB) were trypsinized and proteins extracted in sample buffer containing $62.5 \mathrm{mmol} / \mathrm{L}$ Tris- $\mathrm{HCl}, \mathrm{pH} 6.8$, $2 \%$ SDS, $10 \%$ glycerol, $50 \mathrm{mmol} / \mathrm{L}$ dithiothreitol, and $0.01 \%$ bromophenol blue. ${ }^{24}$ Dissected tissues were immediately frozen in liquid nitrogen and crushed. SMCs and tissues were lyzed in Iso buffer $[9 \mathrm{~mol} / \mathrm{L}$ urea, $1 \%$ Tween 20, $40 \mathrm{mmol} / \mathrm{L}$ Tris, and $162 \mathrm{mmol} / \mathrm{L} \mathrm{1,4-dithioerythritol}$ (DTE) (MP Biomedicals, Irvine, CA)], and protein concentration was determined according to the method of Bradford. ${ }^{25}$ Proteins were separated by SDS-PAGE on $12 \%$ (S100A4 and CaM), 10\% ( $\alpha$-SMA and vimentin), and 5\% (SMMHCs) minigels. They were further stained with Coomassie brilliant blue (R250; Fluka/Sigma-Aldrich). For Western blot analysis, 2 to $15 \mu \mathrm{g}$ of protein was electrophoresed and then was transferred to a nitrocellulose membrane (Protran $0.2 \mu \mathrm{m}$; Schleicher and Schuel, Dassel, Germany) for anti- $\alpha$-SMA, anti-vimentin, and antiSMMHCs or to a $0.45-\mu \mathrm{m}$ polyvinylidene difluoride membrane (Immobilon-P; EMD Millipore Corp., Billerica, MA) for anti-S100A4 and anti-CaM. After incubation with the first antibody, biotinylated goat anti-rabbit (Dako) or anti-mouse (Bio-Rad Laboratories, Richmond, CA) IgGs were used as secondary antibodies. Enhanced chemiluminescence was used for the detection of peroxidase activity (Amersham Biosciences, Sunnyvale, CA). Signals were digitized by means of an Arcus II scanner (Agfa, Mortsel, Belgium) and were analyzed using ImageQuant software (ImageQuant Analysis version 5; Molecular Dynamics, Sunnyvale, CA).

\section{Two-Dimensional PAGE and Protein Identification}

Five distinct cultured SMC populations derived from the DP and three derived from the UP were obtained from six different patients. Moreover, two DP and three UP media tissue samples were obtained from three different patients. To address the issue of individual genetic variability, in two cases the UP and DP media samples were collected from the same patient.

The two-dimensional PAGE assays were performed as previously described ${ }^{13,26,27}$ with minor modifications. Dissected tissues were immediately frozen in liquid nitrogen and crushed. SMCs and tissues were lyzed in Iso buffer (9 mol/L urea, $1 \%$ Tween $20,40 \mathrm{mmol} / \mathrm{L}$ Tris, and 162 $\mathrm{mmol} / \mathrm{L}$ 1,4-dithioerythritol), and protein concentration was determined according to the method of Bradford. ${ }^{25}$

Protein, $150 \mu \mathrm{g}$, was separated in the first dimension with nonlinear immobilized $\mathrm{pH}$ gradient gel strips (Immobiline DryStrip, pH 3 to 10, $18 \mathrm{~cm}$, nonlinear; Amersham Pharmacia Biotech Europe $\mathrm{GmbH}$, Dübendorf, Switzerland) previously rehydrated for at least 12 hours in $7 \mathrm{~mol} / \mathrm{L}$ urea, $2 \mathrm{~mol} / \mathrm{L}$ thiourea, $0.5 \%$ Tween $20,24.5 \mathrm{mmol} / \mathrm{L} \mathrm{1,4-}$ dithioerythritol (MP Biomedicals), 1\% Resolyte (pH 4 to 8) carrier ampholytes (BDH Laboratory Supplies, Poole, UK), $1 \%$ Resolyte (pH 3.5 to 10) carrier ampholytes (BDH Laboratory Supplies), and traces of bromophenol blue. The second dimension was run on $9 \%$ to $16 \%$ acrylamide gels. Gels were either silver stained or subjected to ammoniacal silver staining for subsequent mass spectrometry. To determine the molecular weights and isoelectric points of spots, standards (Bio-Rad Laboratories) were run with cell samples. Silver-stained gels were digitized using a personal laser densitometer (Amersham Biosciences) using LabScan software (Amersham Biosciences). Image analysis was performed using Melanie software version 7.02 (GeneBio, Geneva, Switzerland) ${ }^{28}$ on the visually best three large and two small SMC gels. Albeit gel distortion hampered a complete matching of all protein spots between the gels of the two phenotypes, gels for each phenotype yielded a virtually identical pattern of silver-stained proteins. Spots were detected and matched pairwise. Differentially expressed proteins were identified according to their relative volume.

For statistical analysis estimated through analysis of variance testing, we considered the spots that were exclusively present in the gels of one phenotype and absent in the other. Among the spots exhibiting significance of $P<0.05$, the strongest and most clearly visible one was isolated from ammoniacal silver-stained gels, digested with trypsin, and processed for tandem mass spectrometry analysis using nano liquid chromatography-electrospray ionization tandem mass spectrometry. ${ }^{29}$ The combined Swiss-Prot and TrEMBL database was searched for the tandem mass spectrometrygenerated set of peptides using Mascot software (version 2.2; Matrix Science, London, UK). ${ }^{30}$ A search was performed with a fragment ion mass tolerance of $0.40 \mathrm{Da}$ and a parent ion tolerance of 5.0 parts per million. Iodoacetamide derivative of cysteine was specified in Mascot software as a fixed modification. Deamidation of asparagine, oxidation of methionine, and acetylation of the N-terminus 
were specified in Mascot software as variable modifications. The criteria used for protein identification included number of peptide matches, score of matching probability (Mascot score), and mass accuracy.
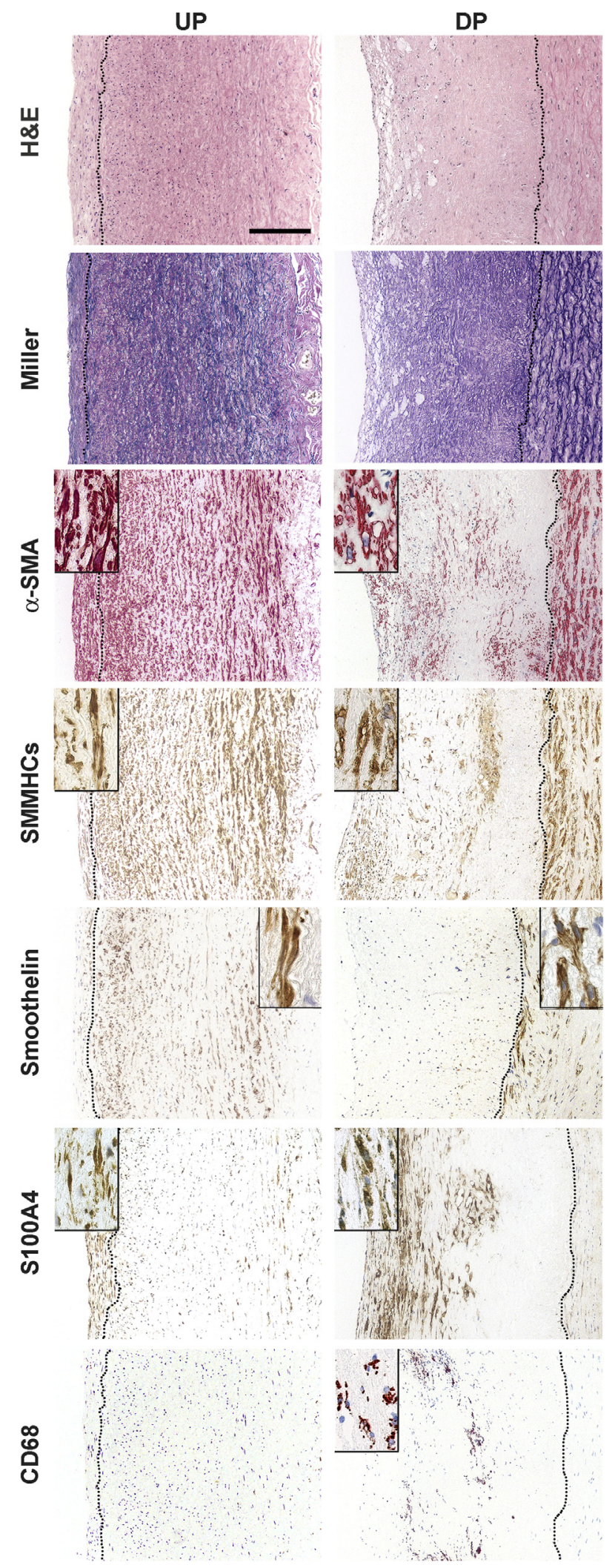

RNA Extraction, Reverse Transcription, and Real-Time Quantitative PCR

Total RNA was prepared from cultured SMCs using TRIzol Reagent (Gibco-Invitrogen). The RNA quality and quantity were determined by Experion RNA StdSens analysis kit (Bio-Rad Laboratories). For each sample, $1 \mu \mathrm{g}$ of total RNA was reverse transcribed using a SuperScript VILO cDNA synthesis kit (Gibco-Invitrogen) according to the manufacturer's recommendations in the following thermal conditions: 10 minutes at $25^{\circ} \mathrm{C}$, then 60 minutes at $42^{\circ} \mathrm{C}$, followed by 5 minutes at $85^{\circ} \mathrm{C}$. Specific primers for human CALM1, CALM2, CALM3, and 18S mRNA were designed as follows (forward and reverse): $5^{\prime}$-TGACTTCCCCGAATTTTTTGAC- $3^{\prime}$ and $5^{\prime}$-TGTCCGTCTCCATCAATATCT-3' ${ }^{\prime}$ covering nucleotides 394 to 609 of CALM1, $5^{\prime}$-AAGTTGATGAAATGATCAGGGAAG- ${ }^{\prime}$ and 5'-TGAAGTCCTAATTACTATACATGCATA-3' covering nucleotides 520 to 739 of CALM2, 5'-ATGATGACTGCAAAGTGAAGGCCC-3' and 5'-GAGCAATCATGCAGCTTGGGACAA-3' covering nucleotides 613 to 797 of CALM 3 , and 5'-GTAACCCGTTGAACCCCATT- ${ }^{\prime}$ and $5^{\prime}$ CCATCCAATCGGTAGTAGCG- $3^{\prime}$ covering nucleotides 1577 to 1729 of 18 S. Real-time quantitative PCR was performed using the Chromo4 real-time PCR detection system (Bio-Rad Laboratories) using SsoFast EvaGreen supermix PCR (Bio-Rad Laboratories). Denaturation was performed at $95^{\circ} \mathrm{C}$ for 30 seconds, then cDNA was amplified for 40 cycles of 5 seconds at $95^{\circ} \mathrm{C}$ and 10 seconds at $60^{\circ} \mathrm{C}$. Each reaction was performed in triplicate. The relative levels of each RNA were calculated by the comparative $\mathrm{C}_{\mathrm{T}}$ method. Results were normalized to amplified $18 \mathrm{~S}$ transcripts in the same samples and are expressed as fold change in small $(n=3)$ compared with large $(n=3)$ SMCs and large SMCs treated with plaque pieces from the $\mathrm{DP}(n=2)$, plaque-derived macrophages $(n=$ $2)$, or plaque-derived CM $(n=1)$ with untreated large SMCs. Each real-time PCR experiment was repeated at least twice.

\section{CaM Inhibition}

CaM inhibition was accomplished with $\mathrm{N}-(6$-aminohexyl)5-chloro-1-naphthalene sulfonamide (W-7; Fluka/SigmaAldrich). To assess cell proliferation, SMCs $(n=2$ for each phenotype) were plated in RPMI 1640 medium supplemented with $10 \%$ FBS at a density of 80 cells $/ \mathrm{mm}^{2}$ with

Figure 1 Histologic and immunohistochemical features of the UP and DP from human CEA specimens. H\&E and Miller staining show that the UP and DP correspond to types III and IV-V lesions, respectively (American Heart Association classification). Immunostaining for $\alpha$-SMA, SMMHCs, smoothelin, S100A4, and CD68 shows that nearly all SMCs in the media of the UP and DP express SMC differentiation markers. Intimal SMCs of the UP and DP expressed $\alpha$-SMA and, to a lesser extent, SMMHCs; they were negative for smoothelin and strongly positive for $\mathrm{S} 100 \mathrm{~A} 4$. $\mathrm{CD} 68$-positive macrophages were present in the lesion of the DP. Insets show the figure in the intimal thickening region except for smoothelin staining, which was taken in the media area. Original magnification, $\times 4$. Dotted lines highlight the internal elastic lamina. The lumen is located on the left side of the images. Scale bar $=200 \mu \mathrm{m}$. 
A

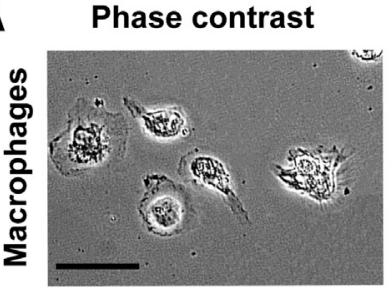

B

Large SMCs from UP media

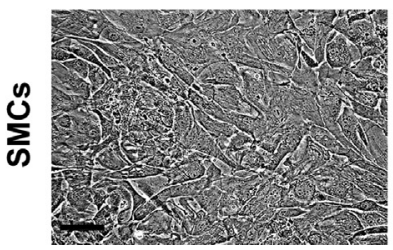

Oil Red 0

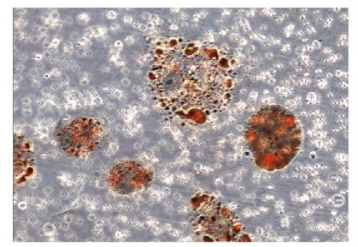

Small SMCs from UP media + plaque

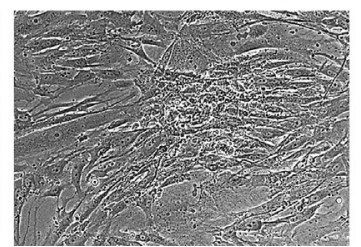

CD68

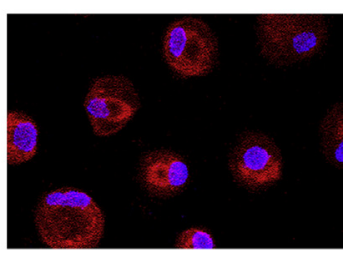

Small SMCs from DP media + plaque

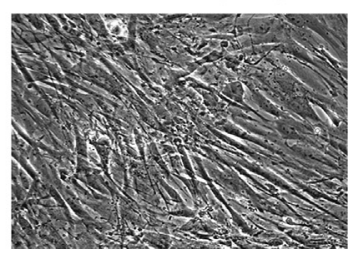

Figure 2 Characterization of macrophagederived foam cells and morphologic features of carotid artery SMC populations. A: Phase-contrast image, Oil Red 0, and CD68 staining showed that macrophages exhibit a foamy cytoplasm with 0il Red 0 -positive inclusions. These cells are positive for $\mathrm{CD} 68$, a marker of the macrophage lineage. The $0.4-\mu \mathrm{m}$ pores of the Transwell membranes are visible in the background as bright spots. B: Phase-contrast images show large SMCs isolated from the media of the UP and small SMCs isolated from the media of the UP and DP co-cultured with plaque. Note the multilayer appearance of small SMCs. Scale bars: $50 \mu \mathrm{m}$ (A); $500 \mu \mathrm{m}$ (B). or without $10 \mu \mathrm{mol} / \mathrm{L} \mathrm{W}-7$ for 72 hours; SMCs were incubated with $10^{-5} \mathrm{~mol} / \mathrm{L} \mathrm{BrdU}$ (Fluka/Sigma-Aldrich) for an additional 20 hours of treatment. Experiments were performed in triplicate. To evaluate the effect of W-7 on phenotypic transition, large SMCs $(n=4)$ were plated in RPMI 1640 medium supplemented with 10\% FBS at a density of 80 cells $/ \mathrm{mm}^{2}$ with or without plaque-derived $\mathrm{CM}$ combined with or without $10 \mu \mathrm{mol} / \mathrm{L} \mathrm{W}-7$ for 3 (immunofluorescence staining and Western blot analysis) or 7 (cell proliferation) days.

\section{Statistical Analysis}

Results are shown as means \pm SEM. Comparisons between the treated and control groups were analyzed using Student's $t$-test. Multiple comparisons were performed by analysis of variance. Differences were considered statistically significant at $P<0.05$.

\section{Results}

\section{Characterization of Arterial Tissues}

All CEA specimens $(n=15)$ included the entire intima and the media comprising the layers up to the external elastic lamina. UP specimens $(n=5)$ exhibited a media and a thin thickened intima, consistent with American Heart Association type III intermediate lesions (Figure 1). ${ }^{31} \mathrm{DP}(n=10)$ specimens exhibited a media underlying an atheromatous plaque corresponding to American Heart Association type IV-V atheroma-fibroatheroma. ${ }^{31}$ Miller staining showed the typical architecture of a muscular artery with abundant elastic fibers in the media. Nearly all SMCs of UP and DP media were positive for three well-accepted SMC differentiation markers: $\alpha$-SMA, SMMHCs, and smoothelin. In the UP, most of the smoothelin-positive SMCs were located in the inner and outer portions of the media; in the DP, instead, in the inner portion of the media. Media SMCs were negative for S100A4, a newly described marker of intimal SMCs, ${ }^{13}$ and for inflammatory cell markers. SMCs of the UP intimal thickening and of the DP plaque stained positively for $\alpha$ SMA and to a lesser extent for SMMHCs; they were negative for smoothelin. In the DP plaque, CD68-positive macrophages were essentially located at the shoulder region, whereas CD79 was absent and CD3-positive lymphocytes were scarcely detected (data not shown). S100A4 was strongly expressed in UP and DP intimal SMCs.

\section{Isolation and Characterization of Plaque-Derived Macrophages}

Many round cells (50 to 100 for each explant) emerged from the DP plaque explants 24 to 48 hours after plating $(n=3)$ (Figure 2A). They displayed a flattened appearance and a foamy cytoplasm with refractile Oil Red $\mathrm{O}$-positive inclusions. ${ }^{32}$ Positive staining for CD68 identified them as macrophage-derived foam cells. After 4 to 14 days of culture, approximately $25 \%$ acquired an elongated shape (data not shown). Macrophages remained viable in culture for 3 to 4 weeks, and then they started detaching.

\section{Human Carotid Artery Yields Two Distinct SMC Phenotypes}

The UP media ( $n=25$ ) cultured alone gave rise to SMCs exhibiting a large phenotype with a flat, polygonal morphology and usually a monolayer growth pattern at confluence. The DP media $(n=23)$ cultured alone did not produce SMCs. In contrast, the DP media co-cultured with plaque or plaquederived macrophages or cultured with plaque-derived CM produced small SMCs that displayed an elongated appearance and grew in multilayers at confluence (Figure 2B). Moreover, small SMCs were isolated when the UP media was co-cultured with plaque or plaque-derived macrophages or cultured with plaque-derived CM (Figure 2B). Large SMCs exhibited a bigger means \pm SEM area than small SMCs $(n=4$ for each phenotype; $9305 \pm 1138 \mu^{2}$ versus $5052 \pm 688 \mu^{2}$, respectively; $P<0.001)$. The mean proportion of explants 
A

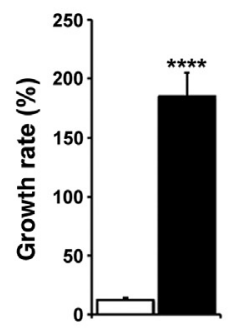

B

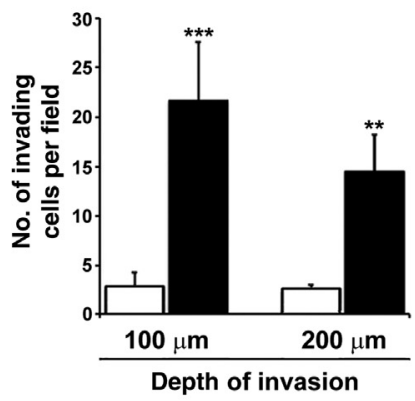

C
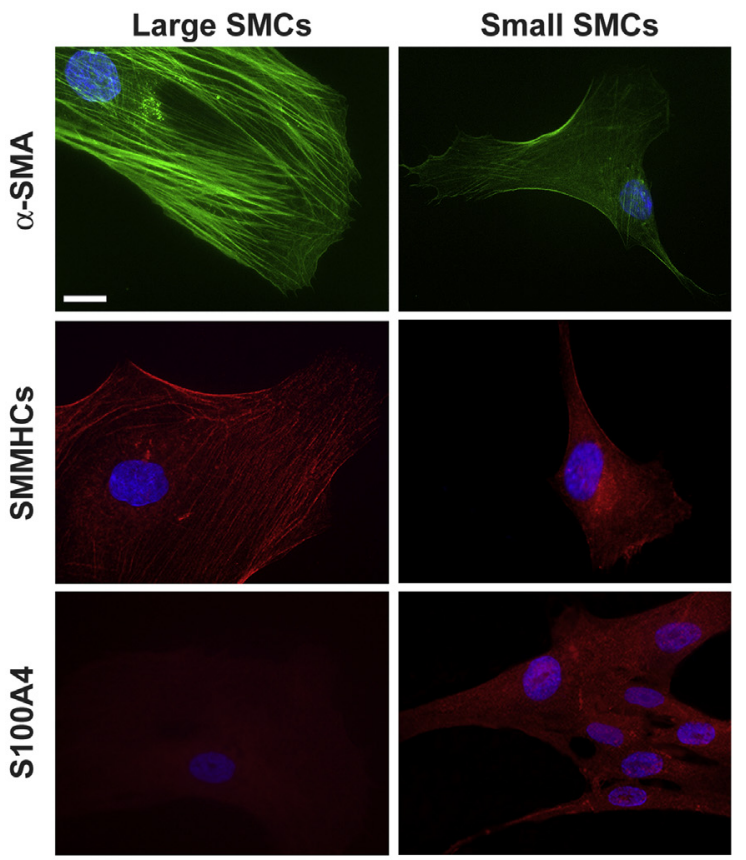

D

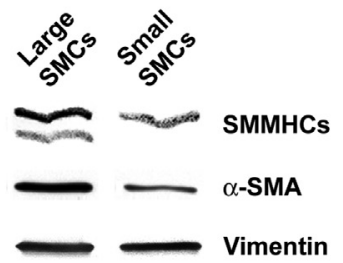

Figure 3 Proliferative (A) and invasive (B) activities of carotid artery SMC populations. Bar graphs show that small SMCs (black bars) proliferate and migrate more actively than large SMCs (white bars). C: Cytoskeletal protein and S100A4 expression in carotid artery SMC populations Immunofluorescence staining of large and small SMCs for $\alpha$-SMA (in green), SMMHCs (in red), and S100A4 (in red). Nuclei were stained in blue by DAPI. Note that $\alpha$-SMA was located in stress fibers in large SMCs, whereas it showed a more diffuse pattern in small SMCs. Large SMCs display the typical interrupted distribution of SMMHCs. S100A4 is diffusely expressed in small SMCs. Scale bar $=20 \mu \mathrm{m}$. D: Representative immunoblot showing the expression of $\alpha$-SMA and SMMHCs in large and small SMCs. Both SMC differentiation markers were weakly expressed in small SMCs compared with large SMCs. Vimentin was used as a loading control. Data are given as means \pm SEM. ${ }^{* *} P<0.01,{ }^{* * *} P<0.001$, and $* * * P<0.0001$ in small versus large SMCs.

yielding SMC outgrowth was higher for the UP media (approximately $75 \%$ of the explants) than for the DP media (approximately $25 \%$ of the explants). Although large SMCs started to grow from the explants more rapidly than did small
SMCs (at 5 to 7 and 6 to 10 days, respectively), small SMCs reached confluence after 10 to 14 days from the appearance of the first cells, whereas large SMCs formed subconfluent monolayers at 14 to 21 days. Both phenotypes remained stable after several passages (up to the 10th passage).

\section{Large and Small SMCs Exhibit Distinct Biological and Biochemical Features}

After primary culture, small SMCs grew rapidly and continued to replicate after reaching confluence, whereas large SMCs grew slowly and stopped growing at subconfluence. The proliferation rate of small SMCs was higher than that of large SMCs $(n=4$ for each phenotype; mean \pm SEM percentage growth rate at 7 days, $185.0 \% \pm 19.8 \%$ and $12.4 \% \pm 1.0 \%$, respectively; $P<0.0001$ ) (Figure 3A).

The collagen gel invasion assay showed that the number of invading cells at each 50- $\mu \mathrm{m}$ interval from the top $(0 \mu \mathrm{m})$ to $700 \mu \mathrm{m}$ deep was higher for small SMCs $(n=3)$ than for large SMCs $(n=4)$, with the highest mean \pm SEM differences at $100 \mu \mathrm{m}$ deep $(21.4 \pm 6.7$ versus $3.3 \pm 1.4$, respectively; $P<0.001)$ and $200 \mu \mathrm{m}$ deep $(14.3 \pm 3.7$ versus $2.8 \pm 0.8$, respectively; $P<0.01$ ) (Figure 3B). Consistently, the mean \pm SEM maximal depth of invasion was higher for small SMCs versus large SMCs (714.3 \pm 45 $\mu \mathrm{m}$ versus $260.0 \pm 44 \mu \mathrm{m} ; P<0.0001)$.

Most large SMCs $(n=13$; mean \pm SEM, $82.9 \% \pm$ $8.8 \%)$ displayed a typical $\alpha$-SMA staining pattern with strongly positive stress fibers (Figure $3 \mathrm{C}$ ); the mean \pm SEM number of $\alpha$-SMA-positive cells $(68.8 \% \pm 12.1 \%)$ and the intensity of the staining was lower in small SMCs $(n=14$; compared with large SMCs. Although a mean \pm SEM of $83.8 \% \pm 3.1 \%$ of large SMCs expressed SMMHCs with the classic interrupted staining pattern at the cell periphery, only a mean \pm SEM of $17.5 \% \pm 2.7 \%(P<0.0001)$ of small SMCs were positive for SMMHCs and displayed less intense, more diffuse staining. Smoothelin was not detected in either SMC population. Large SMCs stained negative for S100A4, whereas small SMCs expressed S100A4 (mean \pm SEM, $42.0 \% \pm 6.6 \%$ ) with a diffuse staining pattern.

Immunoblotting results confirmed immunofluorescence analysis (Figure 3D). In small SMCs, $\alpha$-SMA $(n=4$ for each population) and SMMHC ( $n=3$ for each population) mean \pm SEM content was decreased to $43.7 \% \pm 6.3 \%(P<0.0001)$ and $33.0 \% \pm 6.9 \%(P<0.0001)$, respectively, compared with large SMCs. SMMHC types 1 and 2 were expressed in large SMCs, whereas only SMMHC type 1 was present in small SMCs. Similar results were obtained after loading according to cell number (data not shown).

Plaque, Plaque-Derived Macrophages, and PlaqueDerived CM Induce SMC Phenotypic Transition

When large SMCs were co-cultured with plaque pieces from the DP $(n=3)$ or plaque-derived macrophages $(n=$ 2 ), they grew more rapidly compared with the control 

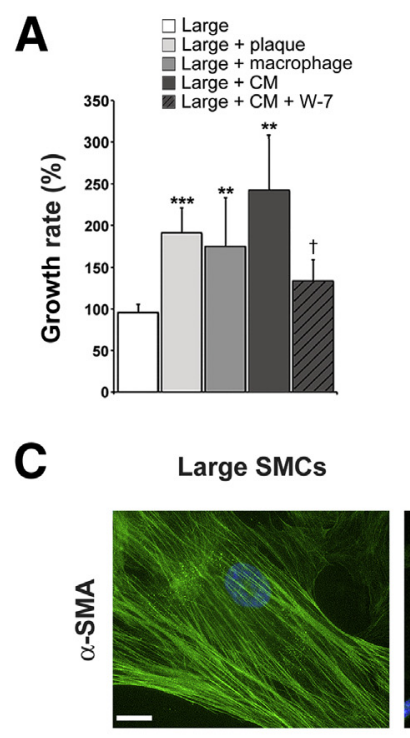

Large SMCs
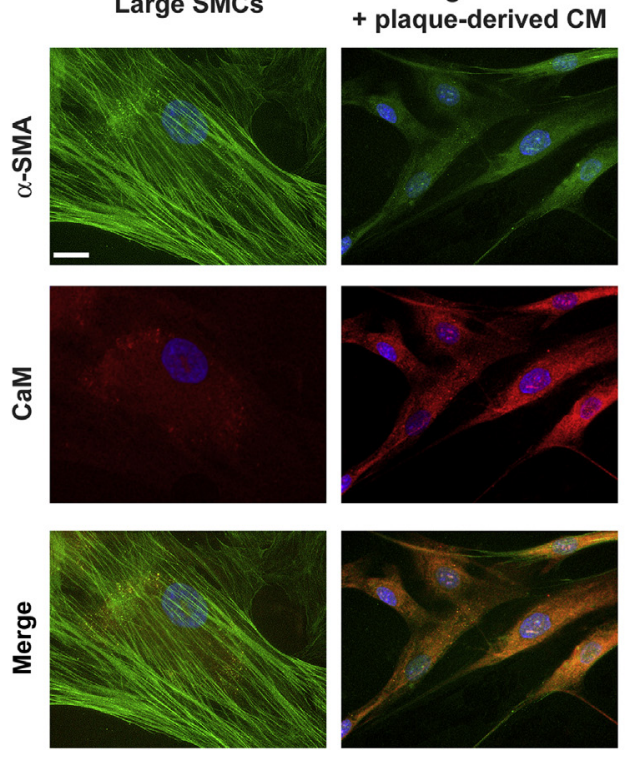

B

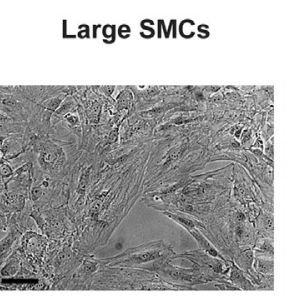

Large SMCs

+ plaque-derived CM

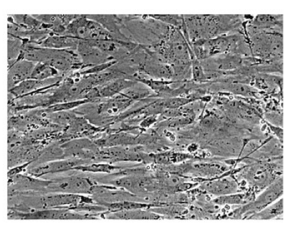

Figure 4 SMC phenotypic transition. A: Proliferative activity of large SMCs in control conditions after co-culture with plaque pieces from the DP and plaque-derived macrophages after culture with plaque-derived $\mathrm{CM}$ and after cotreatment with W-7. B: Phase-contrast images of large SMCs in control conditions and after culture with plaque-derived CM. C: Immunofluorescence staining of large SMCs in control conditions and after culture with plaque-derived CM for $\alpha$-SMA (in green) and CaM (in red). Double-labeled structures appear in yellow on merged images. Nuclei are stained in blue with DAPI. D: Quantification of typical $\alpha$-SMA- and CaM-positive large SMCs in control conditions, after co-culture with plaque pieces from the DP and plaque-derived macrophages, after culture with plaque-derived $\mathrm{CM}$, and after co-treatment with W-7. E: Representative immunoblot showing the expression of $\alpha$-SMA and CaM in large SMCs in control conditions, after culture with plaque-derived CM, and after cotreatment with $W-7$. Data are given as means \pm SEM. ${ }^{* *} P<0.01,{ }^{* * *} P<0.001$ compared with control. ${ }^{\dagger} P<0.05$ compared with culture with plaque-derived CM. condition (mean \pm SEM percentage growth rate at 7 days: $193.2 \% \pm 26.9 \%, P<0.001$, and $177.3 \% \pm 61.4 \%, P<$ 0.01 , respectively) (Figure 4A). Similar results were obtained when large SMCs were cultured with plaque-derived $\mathrm{CM}(n=3$; mean \pm SEM percentage growth rate at 7 days, $234.3 \% \pm 64.4 \% ; P<0.01$ ) (Figure $4 \mathrm{~A}$ ). The acquisition of a highly proliferative phenotype was associated with transition from the flat, polygonal morphology to the small, elongated appearance typical of small SMCs (Figure 4B); this transition occurred in most large SMCs.

On immunofluorescence staining (Figure 4C), there was a significant reduction in the number of large SMCs exhibiting typical $\alpha$-SMA-positive stress fibers after co-culture with plaque pieces from the $\mathrm{DP}(n=2 ;<0.5 \%)$ and plaque-derived macrophages $(n=1 ; 18.8 \%)$ or culture with plaque-derived CM $(n=4$; mean \pm SEM, $15.9 \% \pm$ 4.7\%) compared with control conditions $(n=7$; mean \pm SEM, 59.3\% $\pm 7.7 \% ; P<0.01$ in all conditions) (Figure 4D). This was associated with a concomitant increase in the percentage of SMCs displaying diffuse and very low $\alpha$-SMA staining after co-culture with plaque pieces from the DP $(>99.5 \%)$ or plaque-derived macrophages $(81.2 \%)$ or after culture with plaque-derived CM (mean \pm SEM, $84.1 \pm 4.0 \%$ ) compared with control conditions (mean $\pm \mathrm{SEM}, 40.7 \% \pm 7.7 \% ; P<0.01$ in all conditions). Immunoblotting (Figure 4E) confirmed that after co-culture with plaque-derived macrophages or culture with plaque-derived CM $(n=5)$, mean \pm SEM $\alpha$-SMA content was decreased to $70.3 \% \pm 9.7 \%$ compared with the control condition $(P<0.01)$.

\section{Large and Small SMCs Exhibit Distinct Protein Patterns}

The relative volume of spots, which is directly related to the concentration of the corresponding protein, was computed in the $\mathrm{pH}$ range of 3 to 10 . We quantified a mean \pm SEM of $1234 \pm 99$ spots in large SMC gels and $1401 \pm 110$ spots in small SMC gels (Figure 5A). Pairwise spot matching reached 68\% in the group of large SMCs and 78\% in the group of small SMCs, thus demonstrating homogeneity of the protein expression patterns for each cell phenotype even among different patients.

An arbitrary number was assigned to each spot. Fifteen differentially expressed spots were found $(P<0.01$, analysis of variance test), among them 5 spots were more expressed in large and 10 in small SMC gels (Figure 5A). Moreover, 407 spots were exclusively present in the large and 703 in the small SMC gels. As an example, six in large and nine in small SMC gels exhibiting the highest relative volume associated with a small dispersion in the same group 
A

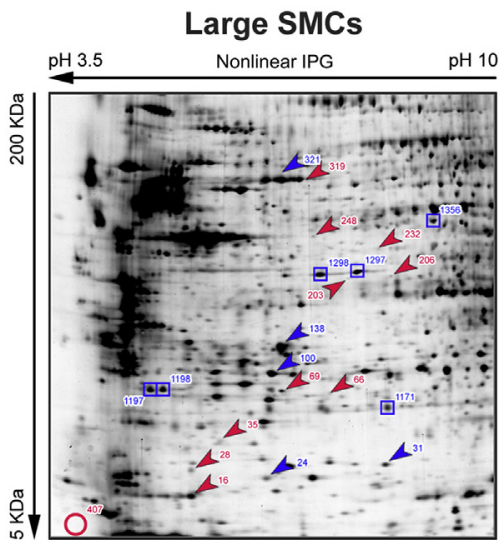

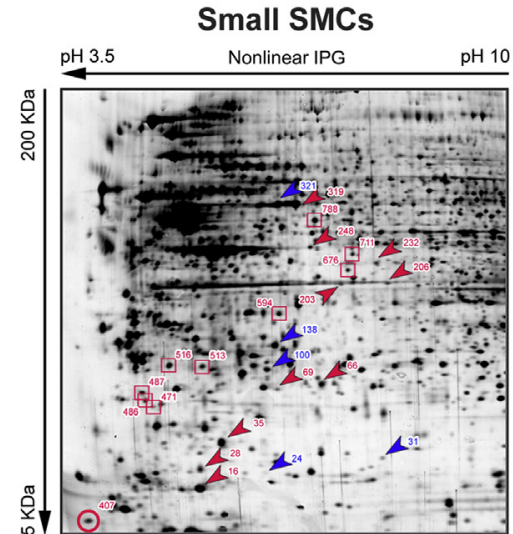

B

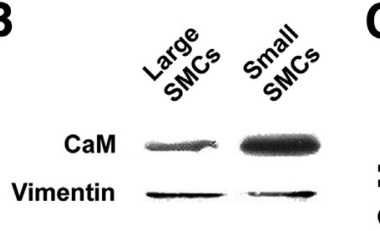

C

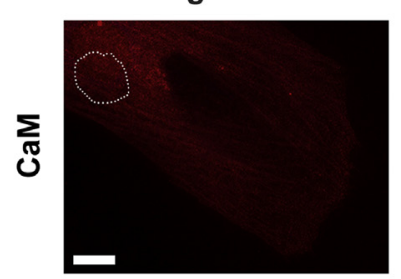

Large SMCs

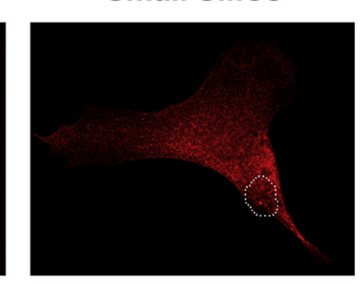

D
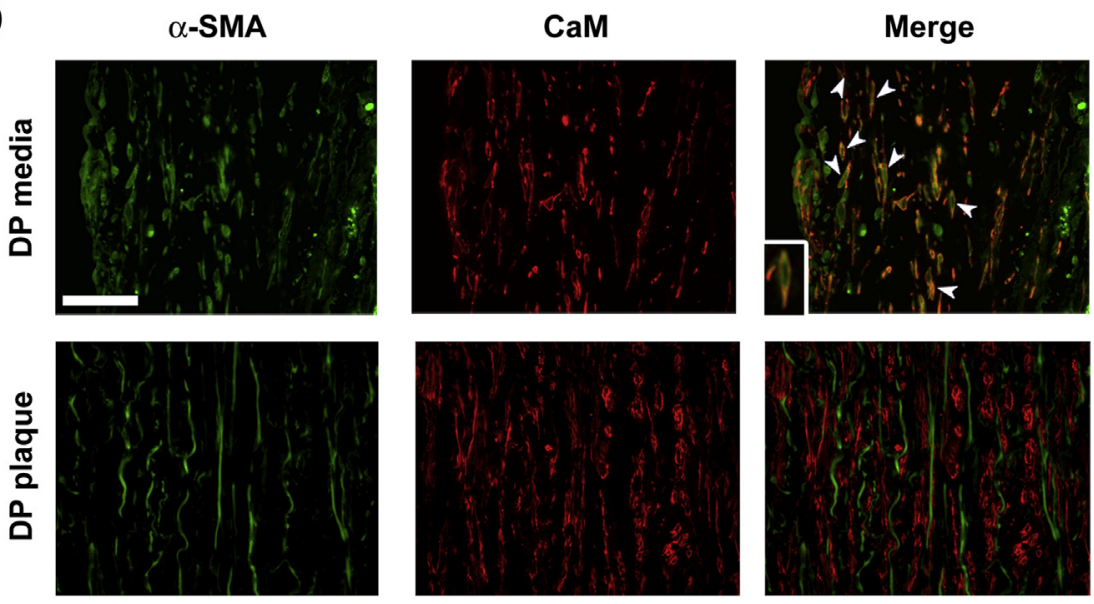

Figure 5 Identification of differentially expressed spots in carotid artery SMC populations. A: Representative silver-stained two-dimensional PAGE gels of large and small SMCs. Spots of higher relative volume in large SMCs compared with small SMCs are indicated by blue arrowheads, whereas spots of higher relative volume in small SMCs compared with large SMCs are indicated by red arrowheads. Spots exclusively present in large SMCs are surrounded by blue squares, and spots present in small SMCs are surrounded by red squares. The CaM spot in the small SMC gel and its migration position in the large SMC gel where it is absent are marked by circles. IPG, immobilized pH gradient. B: Representative immunoblot showing the expression of CaM in large and small SMCs. CaM was markedly expressed in small SMCs compared with large SMCs. Vimentin was used as a loading control. C: Immunofluorescent localization of CaM (in red) in large and small SMCs. The nucleus is depicted by a dotted line. CaM staining was performed as a double staining with the $\alpha$-SMA displayed in Figure 3C (in green). Note stronger CaM expression in small compared with large SMCs. D: Double immunofluorescence staining showing the expression of $\alpha$-SMA (in green) and CaM (in red) and in the atherosclerotic plaque and underlying media of a DP from a human CEA specimen. Double-labeled structures appear in yellow on the merged images. Arrowheads highlight doublelabeled cells. Note that CaM was expressed by $\alpha$ SMA-positive intimal SMCs. The green staining between $\alpha$-SMA-positive cells corresponds to elastic fibers in the media. Inset shows the plaque region. Original magnification: $\times 2$. The vessel lumen is located on the left side of the images. Scale bars: $20 \mu \mathrm{m}$ (C); $50 \mu \mathrm{m}$ (D). of gels and a small coefficient of variation are shown in Figure 5A.

\section{CaM Is Predominantly Expressed in Small SMCs}

Among the spots exclusively present in small gels (five for small and three for large SMCs), spot 407 (Figure 5A) exhibited one of the highest relative volumes. The set of tandem mass spectra obtained for this protein spot was compared with the combined Swiss-Prot and TrEMBL database using Mascotsoftware. The top matching protein was human CaM (Accession No. P62158; length, 149 amino acids; molecular weight, 16,837.59 Da). Six peptides were matched, with a total Mascot score of 191. The molecular weight (16,838 Da) and isoelectric point (4.09) calculated on two-dimensional gels were in agreement with those calculated on two-dimensional maps for HeLa cells and human lymphocytes.

Western blot analysis performed with anti-CaM confirmed the higher expression of CaM in small SMCs $(n=8)$ compared with large SMCs $(n=5)$ : a single band was detected at the expected molecular weight of approximately 17 $\mathrm{kDa}$ (Figure 5B). CaM content represented a mean $\pm \mathrm{SEM}$ of $389.2 \% \pm 117.3 \%(P<0.001)$ in small compared with large SMCs. By means of immunofluorescence staining ( $n=10$ for each phenotype) (Figure 5C), small SMCs showed more intense diffuse cytoplasmic CaM staining than large SMCs.

A high proportion of small SMCs obtained as outgrowth from the UP and DP media co-cultured with plaque pieces from the DP, plaque-derived macrophages, or plaquederived $\mathrm{CM}$ ( $n=8$, in total) was characterized by strong 
A

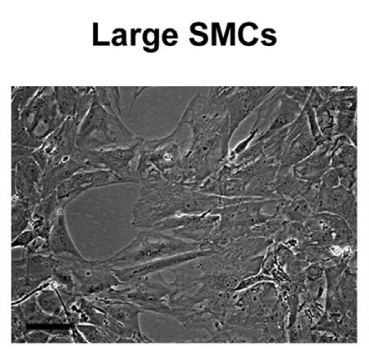

B
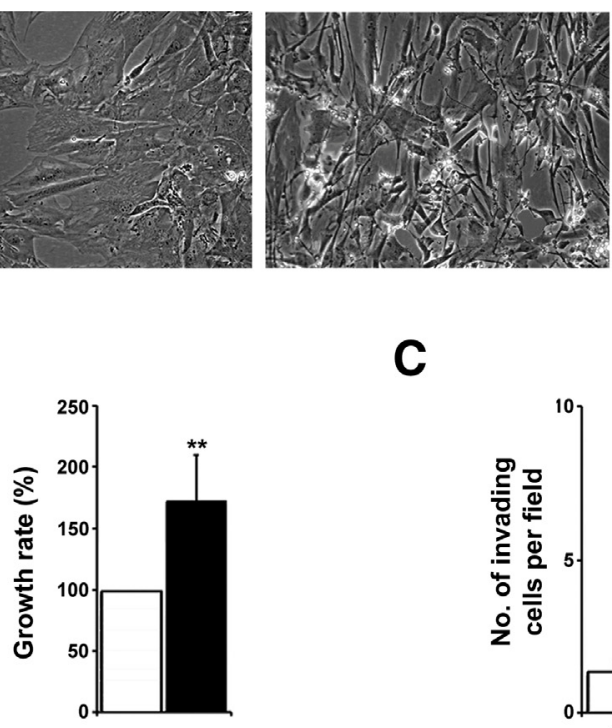
(PDGF-BB withdrawal)

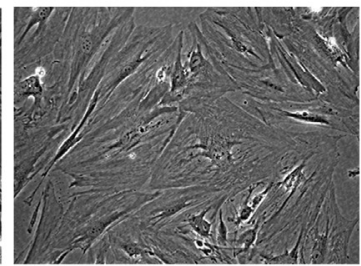

C
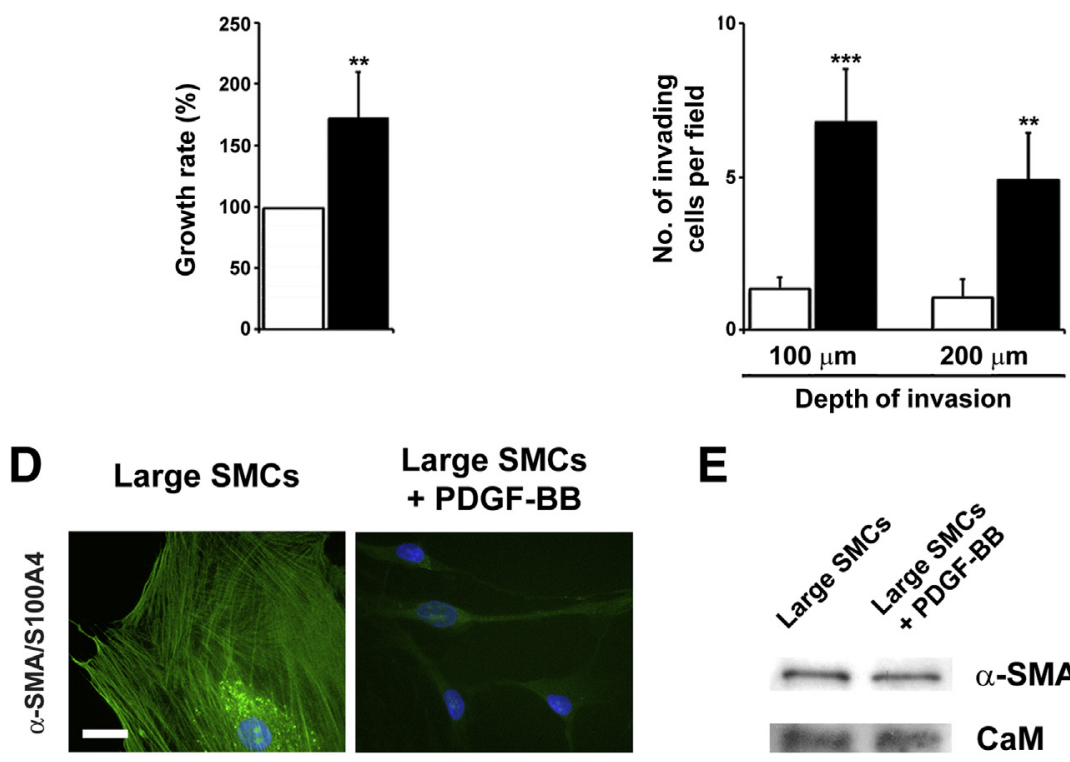

E

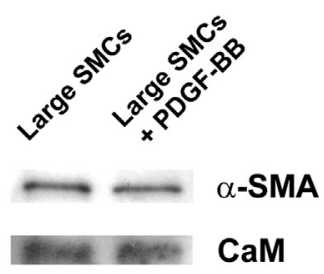

Figure 6 Effect of PDGF-BB on large SMCs. A: Phase-contrast images show large SMCs in control conditions, after treatment with PDGF-BB, and after PDGF-BB withdrawal for 6 days. Proliferative (B) and invasive (C) activities of large SMCs in control conditions and after treatment with PDGFBB. Bar graphs show that large SMCs treated with PDGF-BB (black bars) proliferate and migrate more actively than large SMCs (white bars). D: Immunofluorescence staining of large SMCs in control conditions and after treatment with PDGF-BB for $\alpha$-SMA (in green). Double-labeled structures appear in yellow on merged images. Nuclei were stained in blue with DAPI. E: Representative immunoblot showing the expression of $\alpha$-SMA and CaM in large SMCs in control conditions and after treatment with PDGF-BB. Data are given as means \pm SEM. ${ }^{* *} P<0.01,{ }^{* * *} P<0.001$ in large SMCS treated with PDGF-BB versus large SMCs in control conditions.
CaM-positive staining (mean \pm SEM, $53.0 \% \pm 11.3 \%$; data not shown). In contrast, very few large SMCs growing out from UP explants $(n=1)$ displayed such staining $(<0.5 \%$; data not shown).

The large to small phenotypic transition was associated with a significant increase in the proportion of CaM-positive cells (Figure 4, C and D) after co-culture with plaque pieces from the DP ( $n=2$; mean \pm SEM, $20.2 \% \pm 0.9 \%$ ) or plaquederived macrophages $(n=1 ; 10.2 \%)$ and after culture with plaque-derived CM $(n=4$; mean $\pm \operatorname{SEM}, 29.1 \% \pm 10.2 \%)$ compared with untreated large SMCs $(n=7$; mean \pm SEM, $2.5 \% \pm 0.7 \%, P<0.001$ and $P<0.01)$. Immunoblotting showed that after co-culture with plaque-derived macrophages or culture with plaque-derived CM $(n=3)$, mean \pm SEM $\mathrm{CaM}$ content was increased to $134.2 \% \pm 17.8 \%$ compared with the control condition $(P<0.05)$ (Figure $4 \mathrm{E})$.

\section{PDGF-BB Influences SMC Phenotype and Behavior}

Treatment of large SMCs with PDGF-BB $(n=7)$ resulted in the transition toward the small phenotype characterized by changes in cell morphology (ie, small, elongated cells) (Figure 6A), increased cell proliferation $(n=2$; mean \pm SEM percentage growth rate increase at 7 days, $73.5 \pm 3.8 \%$ compared with untreated large SMCs, $P<0.01$ ) (Figure 6B), and enhanced invasive activity through the collagen gel $(n=$ 4 ; mean \pm SEM: untreated versus treated at $100 \mu \mathrm{m}$ deep, 1.4 \pm 0.4 versus $6.8 \pm 1.7$, respectively; $P<0.001$; untreated versus treated at $200 \mu \mathrm{m}$ deep, $1.1 \pm 0.6$ versus $4.9 \pm 1.5$, respectively; $P<0.01$ ) (Figure $6 \mathrm{C}$ ). The effect on cell morphology was reversed when PDGF-BB was withdrawn for 6 days (Figure 6A). In treated cells, these changes were accompanied by a reduction in the number of SMCs displaying typical $\alpha$-SMA-positive stress fibers ( $n=1,7.0 \%$ versus $46.3 \%$ in treated versus untreated large SMCs). Western blot analysis $(n=2)$ (Figure 6E) showed that CaM expression was unchanged in PDGF-BB-treated compared with untreated large SMCs.

\section{CaM Inhibition with W-7}

Treatment with W-7 decreased mean \pm SEM BrdU incorporation to $69.2 \% \pm 6.5 \%$ in large SMCs and to $22.9 \% \pm$ $12.6 \%$ in small SMCs compared with control conditions ( $n=2$ for each phenotype; $P<0.05$ and $P<0.01$, respectively). This reduction was significantly stronger in small than in large SMCs $(P<0.05)$.

The large to small phenotypic change induced by plaquederived CM was prevented by co-treatment with W-7. The proliferative effect of plaque-derived CM $(n=3$; mean \pm 
SEM percentage growth rate at 7 days, $234.3 \% \pm 64.4 \%$ ) (Figure 4A) on large SMCs was also prevented by cotreatment with W-7 (mean \pm SEM percentage growth rate at 7 days, $135.4 \% \pm 21.9 \% ; P<0.05)$. Immunofluorescence staining (Figure 4C) showed that the mean \pm SEM percentage of SMCs displaying typical $\alpha$-SMA-positive stress fibers after co-culture with plaque-derived CM $(n=4$; $15.9 \% \pm 4.7 \%$ ) was significantly increased by co-treatment with W-7 $(n=2 ; 37.2 \% \pm 7.2 \% ; P<0.05)$ (Figure 4D), whereas the mean \pm SEM percentage of SMCs displaying diffuse and/or practically negative $\alpha$-SMA staining after coculture with plaque-derived CM $(84.1 \% \pm 4.0 \%)$ was decreased by co-treatment with $\mathrm{W}-7(62.8 \% \pm 7.2 \% ; P<$ $0.05)$. This was not associated with a significant increase in $\alpha$-SMA content (Figure 4E). The proportion of CaMpositive cells was unchanged (Figure $4 \mathrm{D}$ ); this result was confirmed by immunoblotting (Figure 4E).

\section{CaM Is Expressed in SMCs of Human Atherosclerotic Lesions}

Double immunofluorescence staining (Figure 5D) performed with anti-CaM and anti- $\alpha$-SMA on DP specimens showed that $\mathrm{CaM}$ was expressed in a portion of plaque SMCs located toward the lumen. CaM was not detected in the media of the DP. $\alpha$-SMA staining showed that CaMpositive cells were SMCs.

\section{CALM mRNA Expression}

The analysis of mRNA by quantitative PCR indicated a level of CALM1, CALM2, and CALM3 similar in small $(n=3)$ and large $(n=3)$ SMC populations (mean \pm SEM fold change: $1.63 \pm 0.87,1.93 \pm 0.27$, and $1.66 \pm 0.29$, respectively). The large to small phenotypic transition was not associated with significant differences in the mean \pm SEM fold change of CALM1, CALM2, and CALM3 mRNA expression after co-culture with plaque pieces from the $\mathrm{DP}(n=2 ; 0.87 \pm 0.15,1.29 \pm 0.33$, and $1.01 \pm 0.14$, respectively) or plaque-derived macrophages $(n=2$; $0.61 \pm 0.23,1.10 \pm 0.79$, and $1.25 \pm 0.25$, respectively) and after culture with plaque-derived CM $(n=1 ; 1.43$, 1.24 , and 1.49 , respectively) compared with untreated large SMCs $(n=3)$.

\section{Discussion}

The present study establishes the conditions to reproducibly isolate two distinct SMC populations, small and large, from human CEA samples. We also show that macrophage-derived foam cells from plaque promote selective migration from the media of one of these populations, small SMCs, which exhibit features typical of the atheroma-prone phenotype. ${ }^{12}$ The concept that SMCs in the media are heterogeneous and that a predisposed SMC subpopulation is prone to accumulate into the intima is based on the observations by Benditt and
Benditt, ${ }^{5}$ further confirmed by other authors. ${ }^{6,33}$ SMC heterogeneity has been studied in many experimental models $^{7-11}$ and suggested in humans in several situations. ${ }^{15-18}$ Moreover, a clonal SMC subpopulation exhibiting features compatible with the atheroma-prone phenotype has been isolated from the undiseased mammary artery. ${ }^{19}$ The conditions required for cell population isolation are of interest: large SMCs can be cultured only from the UP media, suggesting that the DP media has lost at least a proportion of efficient precursors, corresponding to the well-established media atrophy, ${ }^{34}$ and small SMCs can be recovered from UP and DP media but only on co-culture with macrophage-derived foam cells present in the plaque or culture with plaque-derived $\mathrm{CM}$ supporting the pivotal role of macrophages in SMC recruitment during plaque formation. ${ }^{2,3}$ Yet, unknown factors, such as cytokines ${ }^{2,3}$ and oxidized low-density lipoproteins, ${ }^{4,35}$ released by macrophages and/or foam cells may be responsible for SMC migration; these observations indicate that their action is exerted on a particular SMC population. The same factors capable of promoting the selective recruitment of small SMCs from the media can induce the large to small phenotypic transition.

Large SMCs exhibit many biological features similar to those described for rat aorta ${ }^{9}$ and porcine coronary artery ${ }^{11}$ SMCs, referred to as spindle-shaped SMCs, whereas small SMCs resemble rat epithelioid ${ }^{9}$ and porcine rhomboid ${ }^{11}$ SMCs; in addition, small SMCs express S100A4, a marker recently described in pig rhomboid SMCs, ${ }^{13}$ thus strengthening the previous observation that S100A4 is a marker of human atheromatous and restenotic SMCs. Some differences, however, exist between small SMCs and the atheroma-prone SMC subpopulations described in other species, such as rat, pig, and cow: rat epithelioid, porcine rhomboid, and cow epithelioid/rhomboid SMCs are cobblestone and grow as a monolayer at confluence, whereas human small SMCs are fusiform, with a hill-andvalley growth pattern. A similar phenotype was described in human SMC populations isolated from the media of aorta and renal artery by Björkerud. ${ }^{16}$

Comparison of the two cell phenotypes by means of proteomics, an approach rarely exploited in profiling human SMCs ${ }^{36}$ showed that $\mathrm{CaM}$ is overexpressed by small SMCs in vitro; furthermore, $\mathrm{CaM}$ is already present in small SMCs as they outgrow from the media and is strongly expressed by a subpopulation of SMCs in the carotid artery plaque.

Mammal CaM is encoded by three genes (CALMI, $C A L M 2$, and $C A L M 3$ ) at different chromosomal locations. The different transcripts can provide control of the spatial and temporal availability of $\mathrm{CaM}$. All three $\mathrm{CaM}$ genes were expressed in SMCs, but CaM overexpression in small SMCs was not significantly associated with an increased amount of transcripts from any of the three genes, thus suggesting that the different levels of $\mathrm{CaM}$ are the result of posttranscriptional mechanisms. ${ }^{37}$

$\mathrm{CaM}$ is a small acidic four EF-hand calcium-binding protein ubiquitous in eukaryotes, where it represents the 
main calcium sensor. ${ }^{38}$ Increased intracellular calcium activates $\mathrm{CaM}$, which, in turn, regulates different protein targets (CaM-binding proteins), finally affecting many cellular events (eg, cytoskeletal organization, muscle contraction, gene regulation, and cell growth). ${ }^{39}$ The selective activation of CaM-binding proteins relies on $\mathrm{CaM}$ concentration and the number of bound calcium ions. ${ }^{38,39}$ The crucial role of calcium/CaM signaling in regulation of the cell cycle is well-established. ${ }^{40}$ This has also been demonstrated in SMCs. ${ }^{41,42}$ In particular, it has been recently demonstrated that the $G_{1} / S$ transition is at least partly mediated by calcium/CaM-dependent cyclin E/CDK2 activity. ${ }^{43}$ Consistently, a synthetic peptide-blocking CaMcyclin $\mathrm{E}$ interaction arrests S-phase entry and results in decreased proliferation of human and mouse SMCs. ${ }^{44}$ The role of $\mathrm{CaM}$ in cellular replication has been demonstrated in physiologic and pathologic conditions, including tumorigenesis, a process often accompanied by a rise in the CaM cellular concentration. ${ }^{45}$ In the present study, we show that small SMCs, overexpressing CaM, proliferate more actively than large SMCs and that large to small phenotypic transition is accompanied by a strong increase in $\mathrm{CaM}$ expression not paralleled by significant variations in CALM1, CALM2, and CALM3 mRNA.

Among the different $\mathrm{CaM}$ antagonists commonly used to examine the role of $\mathrm{CaM}$ in cell functioning, ${ }^{46} \mathrm{~W}-7$ was chosen as a most suitable CaM-inhibitory compound because of its high affinity for $\mathrm{CaM}$ and lower cell toxicity compared with other $\mathrm{CaM}$ antagonists (eg, phenothiazines). ${ }^{47}$ It selectively inhibits the activation of CaM targets by binding to its hydrophobic binding pockets. ${ }^{46}$ In addition to its major CaM-dependent effects, W-7 shows a weak inhibitory potential on different calcium/CaM-independent enzymes ${ }^{48}$ including protein kinase $\mathrm{C},{ }^{49}$ that could decrease SMC proliferation. However, the concentrations required to inhibit protein kinase $\mathrm{C}$ are greater than those required to inhibit CaM. ${ }^{46}$ Previous works reported an inhibitory effect of W-7 on DNA synthesis in rat SMCs. ${ }^{41,42,50}$

We showed that inhibition of $\mathrm{CaM}$ with $\mathrm{W}-7$ is associated with a reduction in SMC proliferation that is significantly more important in small compared with large SMCs and prevents the large to small phenotypic transition. These results suggest a functional role of CaM in human atheromaprone SMC behavior.

Although the treatment of large SMCs with PDGF-BB induced a transition toward a small phenotype characterized by increased proliferation and migration and decreased differentiation, $\mathrm{CaM}$ expression was unchanged. This suggests that in the presence of PDGF-BB, CaM overexpression is not necessary to induce SMC proliferation. Among the multiple effects on SMC behavior exerted by PDGF-BB, proliferation relies on different pathways (eg, Ras/Erk, PI3K/Akt), culminating in increased transcription of cyclin D1, increased stabilization of cyclin D1, and suppression of p27Kip1 levels (a cell-cycle regulatory protein). ${ }^{51}$ Moreover, a direct effect of PDGF-BB on CaM synthesis has never been described. It is, therefore, conceivable that $\mathrm{CaM}$ overexpression hallmarks the small SMC phenotype rather than increased SMC proliferation.

In conclusion, these results demonstrate that the media of the human carotid artery contains two phenotypically distinct SMC populations that can be studied in culture: the small SMC population seems to be involved in atherogenesis. Moreover, factor(s) released by plaque macrophage-derived foam cells are necessary for the selective isolation of small SMCs in culture. We also show that $\mathrm{CaM}$ is a marker of small SMCs in vitro and of a subpopulation of intimal SMCs in human carotid artery plaque in vivo. Macrophage-derived foam cells promote the migration of small SMCs from the arterial media and induce a large to small phenotypic transition: these observations strongly indicate a complex interaction between these two cell types. These findings, however, do not yet furnish a clearcut paradigm for understanding whether a preexisting SMC subpopulation in the media is prone to be activated (by hitherto unknown factors released by plaque macrophage-derived foam cells) and to migrate into the intima, or these effects are mainly the expression of SMC plasticity.

Further studies exploring the biological behavior of these populations and the mechanisms of $\mathrm{CaM}$ expression and interaction with target proteins should be instrumental to better understanding the evolution of atherosclerosis and restenosis and possibly to designing therapeutic strategies.

\section{Acknowledgments}

We thank Aman Ahmed-Mohamed, Philippe Henchoz, Antoine Geinoz, and Patrizia Arboit for their technical assistance; Laura Rubbia-Brant for her advice; Dr. Guillaume van Eys (Maastricht University) for his generous gift of smoothelin antibody; the staff of the Operative Unit of Vascular and Endovascular Surgery (S. Anna University-Hospital, Ferrara, Italy); and, particularly, Giulio Gabbiani for his invaluable discussions and suggestions.

\section{References}

1. Faxon DP, Creager MA, Smith SC Jr, Pasternak RC, Olin JW, Bettmann MA, Criqui MH, Milani RV, Loscalzo J, Kaufman JA, Jones DW, Pearce WH: Atherosclerotic Vascular Disease Conference: executive summary: Atherosclerotic Vascular Disease Conference proceeding for healthcare professionals from a special writing group of the American Heart Association. Circulation 2004, 109: 2595-2604

2. Ross R: Atherosclerosis: an inflammatory disease. N Engl J Med 1999, 340:115-126

3. Hansson GK, Libby P: The immune response in atherosclerosis: a double-edged sword. Nat Rev Immunol 2006, 6:508-519

4. Mertens A, Holvoet P: Oxidized LDL and HDL: antagonists in atherothrombosis. FASEB J 2001, 15:2073-2084

5. Benditt EP, Benditt JM: Evidence for a monoclonal origin of human atherosclerotic plaques. Proc Natl Acad Sci U S A 1973, 70: $1753-1756$ 
6. Murry CE, Gipaya CT, Bartosek T, Benditt EP, Schwartz SM: Monoclonality of smooth muscle cells in human atherosclerosis. Am J Pathol 1997, 151:697-705

7. Walker LN, Bowen-Pope DF, Ross R, Reidy MA: Production of platelet-derived growth factor-like molecules by cultured arterial smooth muscle cells accompanies proliferation after arterial injury. Proc Natl Acad Sci U S A 1986, 83:7311-7315

8. Majesky MW, Benditt EP, Schwartz SM: Expression and developmental control of platelet-derived growth factor A-chain and Bchain/Sis genes in rat aortic smooth muscle cells. Proc Natl Acad Sci U S A 1988, 85:1524-1528

9. Bochaton-Piallat ML, Ropraz P, Gabbiani F, Gabbiani G: Phenotypic heterogeneity of rat arterial smooth muscle cell clones: implications for the development of experimental intimal thickening. Arterioscler Thromb Vasc Biol 1996, 16:815-820

10. Frid MG, Aldashev AA, Dempsey EC, Stenmark KR: Smooth muscle cells isolated from discrete compartments of the mature vascular media exhibit unique phenotypes and distinct growth capabilities. Circ Res 1997, 81:940-952

11. Hao H, Ropraz P, Verin V, Camenzind E, Geinoz A, Pepper MS, Gabbiani G, Bochaton-Piallat ML: Heterogeneity of smooth muscle cell populations cultured from pig coronary artery. Arterioscler Thromb Vasc Biol 2002, 22:1093-1099

12. Hao H, Gabbiani G, Bochaton-Piallat ML: Arterial smooth muscle cell heterogeneity: implications for atherosclerosis and restenosis development. Arterioscler Thromb Vasc Biol 2003, 23:1510-1520

13. Brisset AC, Hao H, Camenzind E, Bacchetta M, Geinoz A, Sanchez JC, Chaponnier C, Gabbiani G, Bochaton-Piallat ML: Intimal smooth muscle cells of porcine and human coronary artery express S100A4, a marker of the rhomboid phenotype in vitro. Circ Res 2007, 100:1055-1062

14. Scatena M, Liaw L, Giachelli CM: Osteopontin: a multifunctional molecule regulating chronic inflammation and vascular disease. Arterioscler Thromb Vasc Biol 2007, 27:2302-2309

15. Orekhov AN, Krushinsky AV, Andreeva ER, Repin VS, Smirnov VN: Adult human aortic cells in primary culture: heterogeneity in shape. Heart Vessels 1986, 2:193-201

16. Björkerud S: Cultivated human arterial smooth muscle displays heterogeneous pattern of growth and phenotypic variation. Lab Invest 1985, 53:303-310

17. Babaev VR, Antonov AS, Domogatsky SP, Kazantseva IA: Phenotype related changes of intimal smooth muscle cells from human aorta in primary culture. Atherosclerosis 1992, 96:189-202

18. Llorente-Cortes V, Martinez-Gonzalez J, Badimon L: Differential cholesteryl ester accumulation in two human vascular smooth muscle cell subpopulations exposed to aggregated LDL: effect of PDGFstimulation and HMG-CoA reductase inhibition. Atherosclerosis 1999, 144:335-342

19. Li S, Fan YS, Chow LH, Van Den Diepstraten C, van Der Veer E, Sims SM, Pickering JG: Innate diversity of adult human arterial smooth muscle cells: cloning of distinct subtypes from the internal thoracic artery. Circ Res 2001, 89:517-525

20. North American Symptomatic Carotid Endarterectomy Trial Collaborators: Beneficial effect of carotid endarterectomy in symptomatic patients with high-grade carotid stenosis. N Engl J Med 1991, 325: 445-453

21. Skalli O, Ropraz P, Trzeciak A, Benzonana G, Gillessen D, Gabbiani G: A monoclonal antibody against alpha-smooth muscle actin: a new probe for smooth muscle differentiation. J Cell Biol 1986, 103:2787-2796

22. van der Loop FT, Schaart G, Timmer ED, Ramaekers FC, van Eys GJ: Smoothelin, a novel cytoskeletal protein specific for smooth muscle cells. J Cell Biol 1996, 134:401-411

23. Dugina V, Zwaenepoel I, Gabbiani G, Clement S, Chaponnier C: Beta- and gamma-cytoplasmic actins display distinct distribution and functional diversity. J Cell Sci 2009, 122:2980-2988
24. Laemmli UK: Cleavage of structural proteins during the assembly of the head of bacteriophage T4. Nature 1970, 227:680-685

25. Bradford MM: A rapid and sensitive method for the quantification of microgram quantities of protein utilizing the principle of protein-dye binding. Anal Biochem 1976, 72:248-254

26. Cremona O, Muda M, Appel RD, Frutiger S, Hughes GJ, Hochstrasser DF, Geinoz A, Gabbiani G: Differential protein expression in aortic smooth muscle cells cultured from newborn and aged rats. Exp Cell Res 1995, 217:280-287

27. Neuville P, Geinoz A, Benzonana G, Redard M, Gabbiani F, Ropraz P, Gabbiani G: Cellular retinol-binding protein-1 is expressed by distinct subsets of rat arterial smooth muscle cell in vitro and in vivo. Am J Pathol 1997, 150:509-521

28. Appel RD, Palagi PM, Walther D, Vargas JR, Sanchez JC, Ravier F, Pasquali C, Hochstrasser DF: Melanie II: a third-generation software package for analysis of two-dimensional electrophoresis images, I: features and user interface. Electrophoresis 1997, 18:2724-2734

29. Lescuyer P, Allard L, Zimmermann-Ivol CG, Burgess JA, HughesFrutiger S, Burkhard PR, Sanchez JC, Hochstrasser DF: Identification of post-mortem cerebrospinal fluid proteins as potential biomarkers of ischemia and neurodegeneration. Proteomics 2004, 4:2234-2241

30. Perkins DN, Pappin DJ, Creasy DM, Cottrell JS: Probability-based protein identification by searching sequence databases using mass spectrometry data. Electrophoresis 1999, 20:3551-3567

31. Stary HC: Composition and classification of human atherosclerotic lesions. Virchows Arch A Pathol Anat Histopathol 1992, 421:277-290

32. Kruth HS: Localization of unesterified cholesterol in human atherosclerotic lesions. Demonstration of filipin-positive, oil-red-O-negative particles. Am J Pathol 1984, 114:201-208

33. Pearson TA, Dillman JM, Solex K, Heptinstall RH: Clonal markers in the study of the origin and growth of human atherosclerotic lesions. Circ Res 1978, 43:10-18

34. Hao H, Gabbiani G, Camenzind E, Bacchetta M, Virmani R, Bochaton-Piallat ML: Phenotypic modulation of intima and media smooth muscle cells in fatal cases of coronary artery lesion. Arterioscler Thromb Vasc Biol 2006, 26:326-332

35. Boullier A, Bird DA, Chang MK, Dennis EA, Friedman P, GillotreTaylor K, Horkko S, Palinski W, Quehenberger O, Shaw P, Steinberg D, Terpstra V, Witztum JL: Scavenger receptors, oxidized LDL, and atherosclerosis. Ann N Y Acad Sci 2001, 947:214-222

36. Vivanco F, Martin-Ventura JL, Duran MC, Barderas MG, BlancoColio L, Darde VM, Mas S, Meilhac O, Michel JB, Tunon J, Egido J: Quest for novel cardiovascular biomarkers by proteomic analysis. J Proteome Res 2005, 4:1181-1191

37. Toutenhoofd SL, Strehler EE: The calmodulin multigene family as a unique case of genetic redundancy: multiple levels of regulation to provide spatial and temporal control of calmodulin pools? Cell Calcium 2000, 28:83-96

38. Chin D, Means AR: Calmodulin: a prototypical calcium sensor. Trends Cell Biol 2000, 10:322-328

39. O'Day DH: CaMBOT: profiling and characterizing calmodulinbinding proteins. Cell Signal 2003, 15:347-354

40. Reddy GP, Reed WC, Sheehan E, Sacks DB: Calmodulin-specific monoclonal antibodies inhibit DNA replication in mammalian cells. Biochemistry 1992, 31:10426-10430

41. Thyberg J, Palmberg L: The calcium antagonist nisoldipine and the calmodulin antagonist W-7 synergistically inhibit initiation of DNA synthesis in cultured arterial smooth muscle cells. Biol Cell 1987, 60: 125-132

42. Tomita M, Hirata Y, Takata S, Fujita T: Effects of calciumantagonists and calmodulin inhibitors on DNA synthesis in cultured rat vascular smooth muscle cells. Endocrinol Jpn 1987, 34:313-318

43. Choi J, Chiang A, Taulier N, Gros R, Pirani A, Husain M: A calmodulin-binding site on cyclin E mediates Ca2+-sensitive G1/s transitions in vascular smooth muscle cells. Circ Res 2006, 98: 1273-1281 
44. Hui S, Choi J, Zaidi S, Momen A, Steinbach SK, Sadi AM, Ban K, Husain M: Peptide-mediated disruption of calmodulin-cyclin e interactions inhibits proliferation of vascular smooth muscle cells and neointima formation. Circ Res 2011, 108:1053-1062

45. Hait WN, Lazo JS: Calmodulin: a potential target for cancer chemotherapeutic agents. J Clin Oncol 1986, 4:994-1012

46. Kahl CR, Means AR: Regulation of cell cycle progression by calcium/calmodulin-dependent pathways. Endocr Rev 2003, 24: 719-736

47. Hidaka H, Sasaki Y, Tanaka T, Endo T, Ohno S, Fujii Y, Nagata T: $\mathrm{N}$-(6-aminohexyl)-5-chloro-1-naphthalenesulfonamide, a calmodulin antagonist, inhibits cell proliferation. Proc Natl Acad Sci U S A 1981, 78:4354-4357
48. Schatzman RC, Raynor RL, Kuo JF: N-(6-Aminohexyl)-5-chloro-1naphthalenesulfonamide(W-7), a calmodulin antagonist, also inhibits phospholipid-sensitive calcium-dependent protein kinase. Biochim Biophys Acta 1983, 755:144-147

49. Sugimura M, Sato T, Nakayama W, Morishima Y, Fukunaga K, Omitsu M, Miyamoto E, Shirasaki Y: DY-9760e, a novel calmodulin antagonist with cytoprotective action. Eur J Pharmacol 1997, 336: 99-106

50. Hadrava V, Tremblay J, Hamet P: Intrinsic factors involved in vascular smooth muscle cell proliferation in hypertension. Clin Invest Med 1991, 14:533-544

51. Jones SM, Kazlauskas A: Growth factor-dependent signaling and cell cycle progression. FEBS Lett 2001, 490:110-116 